\title{
Immunohistochemical analysis of MMP-9, MMP-2 and TIMP-1, TIMP-2 expression in the central nervous system following infection with viral and bacterial meningitis
}

\author{
Artur Sulik ${ }^{1}$, Lech Chyczewski² \\ ${ }^{1}$ Department of Pediatric Infectious Diseases, ${ }^{2}$ Department of Clinical Molecular Biology, \\ Medical University of Białystok, Białystok, Poland
}

\begin{abstract}
Matrix metalloproteinases (MMPs) are capable of degrading components of the basal lamina of cerebral vessels, thereby disrupting the blood-brain barrier and inducing leukocyte recruitment. This study provides comprehensive information regarding the cell specificity of matrix metalloproteinases (MMP-2, MMP-9) and their binding tissue inhibitors (TIMP-1, TIMP-2) in the central nervous system during viral and bacterial meningitis. Specifically, we evaluated the immunoreactivity of MMPs and TIMPs in various cell types in brain parenchyma and meninges obtained from autopsy tissues. We found that a higher proportion of endothelial cells were positive for MMP-9 during meningitis when compared to controls. In addition, the immunoreactivity of MMP-9 decreased and the immunoreactivity of TIMP-1 increased in astrocytes upon infection. Furthermore, the results of this study revealed that mononuclear cells were highly immunoreactive for TIMP-1, TIMP-2 and MMP-9 during viral meningitis and that the expression of TIMPs in polymorphonuclear cells was even higher during bacterial meningitis. Taken together the results of this study indicated that the central nervous system resident cells and inflammatory infiltrates contribute to MMPs activity and that the expression patterns vary between cell types and in response to viral and bacterial meningitis.
\end{abstract}

Key words: matrix metalloproteinases, tissue inhibitors of metalloproteinases, meningitis, immunohistochemistry

\section{Introduction}

Central Nervous System (CNS) infections are still an important cause of morbidity and mortality despite newly developed vaccines and continuous improvement in health care. There are more than a dozen species of viruses, bacteria, fungi and parasites capable of causing meningitis or encephalitis. However, disruption of the blood-brain barrier (BBB) and leukocyte infiltration of the CNS are hallmarks of meningitis, regardless of the causative agent. This transmigration of leukocytes into the brain parenchyma occurs via a multi-step process that is mediated by a number of adhesion molecules, selectins and enzymes.

Matrix metalloproteinases (MMPs) are peptides that play an important role in brain injury during infec-

Correspondence: A. Sulik, Dept. of Pediatric Infectious Diseases, Medical University of Bialystok, Waszyngtona 17, 15-274 Bialystok, Poland; tel/fax.: (+4885) 7450685, e-mail: artsul@interia.pl tious and noninfectious inflammation of the central nervous system. This growing family of endopeptidases is capable of degrading components of the extracellular matrix (ECM). In addition to its structural functions, the ECM also serves as a reservoir of substances that modulate cell proliferation, differentiation, migration and death. MMPs have been implicated in several diseases of the central nervous system. For example, increased expression of MMPs has been observed in multiple sclerosis [1], experimental autoimmune encephalomyelitis [2], Alzheimer's disease [3], stroke [4] and meningitis [5]. Numerous studies have provided converging evidence that MMPs play a key role in the pathogenesis of meningitis through their contribution to the breakdown of the BBB and the accumulation of blood-derived immune cells. Gelatinases (MMP-2 and MMP-9) are probably the most extensively studied MMPs in the context of the pathogenesis of meningitis [6-8]. However, the cellular sources of MMP activity in the CNS are not yet clear. It has been suggested that resident cells of the brain, includ- 
ing neurons, astrocytes, microglia and endothelial cells, contribute to the increased expression of MMPs in the CNS during meningitis. It has also been suggested that inflammatory infiltrates such as monocytes, macrophages and granulocytes could possible contribute to this increased expression.

Although it is well known that metalloproteinases are mediators of disease, they also have beneficial functions during the development of CNS and repair following injury [9]. Accordingly, inhibition of metalloproteinase activity leads to alleviation of neuroinflammation. The activity of MMPs is regulated on several levels, including gene transctiption, pro-enzyme activation and inhibition by non-specific or specific enzyme inhibitors. Tissue inhibitors of metalloproteinases (TIMPs) are specific endogenous inhibitors of matrix metalloproteinases that form 1:1 enzymeinhibitor complexes. Currently, there are four known TIMPs, each of which differs in its affinity for specific metalloproteinases. The balance between MMPs and TIMPs regulates extracellular matrix remodeling during the pathogenesis of disease. Furthermore, TIMPs are not only protease inhibitors, but also appear to be important signal transducers [10]. Finally, it is believed that astrocytes produce the majority of TIMPs in the central nervous system.

In the present study, we examined the immunoreactivity of gelatinases (MMP-2 and MMP-9) and two TIMPs (TIMP-1 and TIMP-2) in autopsy brain tissue obtained from patients who were afflicted with purulent and lymphocytic viral CNS infections. We also attempted to determine potential cellular sources of their activity in the central nervous system.

\section{Materials and methods}

Tissue samples. Autopsy brain tissue samples (postmortem delay range 12-16 hours) taken from cortex and subsubcortical white matter from five patients diagnosed with lymphocytic viral meningitis (aged 48-68 years), five patients diagnosed with purulent meningoencephalitis (aged 42-71 years) and five patients without any known abnormalities of the CNS (aged 40-73 years; cause of death: myocardial infarction-3, pneumonia-2) were evaluated in this study. The ratio of male to female was $2: 3$ in all investigated groups. All of the clinical diagnoses of CNS infection were confirmed by histopathological microscopic examination. This study protocol was approved by Ethics Committee of the University.

Immunohistochemistry. Paraffin-embedded sections with a width of $5 \mu \mathrm{m}$ were mounted on glass slides and then deparaffinized with xylene and a series of ethanol. The sections were then treated with $0.5 \%$ hydrogen peroxide in methanol for 10 minutes to block the endogenous peroxidase activity, after which they were washed in tap water. A high temperature antigen unmasking technique was then applied to all sections. Next, the sections were placed in normal serum for 10 minutes and then incubated for 60 minutes with primary antibodies (mouse monoclonal anybodies, Novocastra, UK) specific to MMP-2 (dilution 1:40), MMP-9 (dilution 1:40), TIMP-1 (dilution 1:75) or TIMP-2 (dilution 1:40). For negative controls, the primary antibodies were omitted.
The avidin-biotin technique was then employed to stain the samples. In this technique, a biotinylated secondary antibody reacts with several peroxidase-conjugated streptavidin molecules (Dako Cytomation LSAB+ System). Briefly, the sections were rinsed in TBS buffer, after which they were incubated with biotinylated link antibody and peroxidase-labeled streptavidin. The immunoreactivity was then visualized by incubating the samples in 3,3'-diaminobenzedine. Finally, the slides were counterstained with hematoxilin.

All sections were then evaluated by an experienced pathologist. Immunoreactivity was assessed using a semi-quantitative score, with 0 indicating that the sample was not immunoreactive and 1, 2 and 3 indicating weak, moderate and intense immunoreactivity, respectively.

\section{Results}

To identify potential cellular sources of MMP and TIMP activity in the CNS, we performed an in-depth analysis of their immunoreativity in CNS endothelial cells, astrocytes, neurons and inflammatory infiltrates throughout brain parenchyma obtained from meningitis patients and then compared the results to those obtained when control samples were analyzed (Fig. 1). In addition, infiltrating mononuclear or polymorphonuclear cells were also assessed to determine if MMP or TIMP positive signals occurred in thickened meninges during meningitis (Fig. 2).

MMP-9 was immunolocalized in the endothelial cells from normal controls (Fig. 3a); however, the expression increased in cells obtained from patients that had meningitis of a viral origin (Fig. 3b). This increase in expression was even more apparent in samples obtained from patients with bacterial meningitis. Conversely, MMP-9 immunoreactivity decreased in astrocytes obtained from patients who had viral and bacterial meningitis when compared to controls, whereas TIMP-1 expression increased upon meningitis (Fig. 3c, d). No distinct changes in MMP-2 immunoreactivity (Fig. 3e) were observed in response to meningitis in any of the cell types evaluated in this study.

Interestingly, the neurons were consistently found to be positive for gelatinases and TIMPs in the control group and both meningitis groups. This finding indicates that this type of cell may be an important source of MMP and TIMP activity in both healthy individuals and in individuals with meningitis (Fig. 3f, g).

Image analysis revealed strong MMP-9, TIMP-1 and TIMP-2 positive signals in leukocyte infiltrates along the thickened meninges in samples obtained from patients with viral and bacterial meningitis. Furthermore, MMP-9 (Fig. 3h) and TIMPs immunoreactivity appeared to be greater in samples from patients that were afflicted with bacterial infection than in samples from patients who had viral meningitis.

\section{Discussion}

This study examined the immunoreactivity of matrix metalloproteinases (MMP-9 and MMP-2) and their tis- 


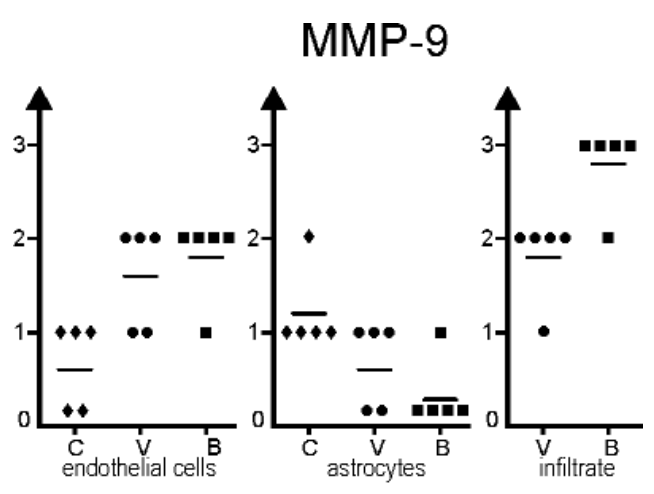

MMP-2

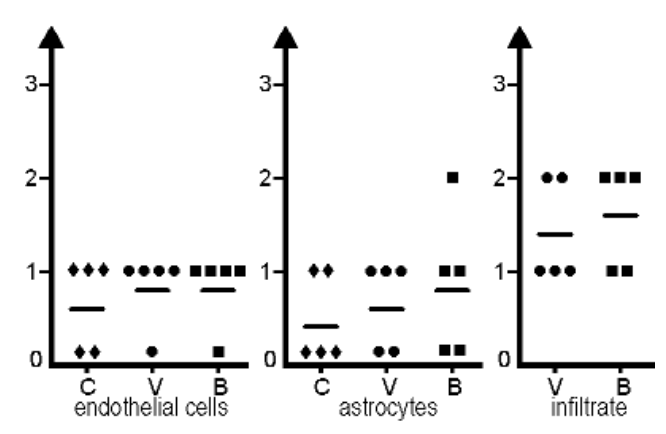

\section{TIMP-1}

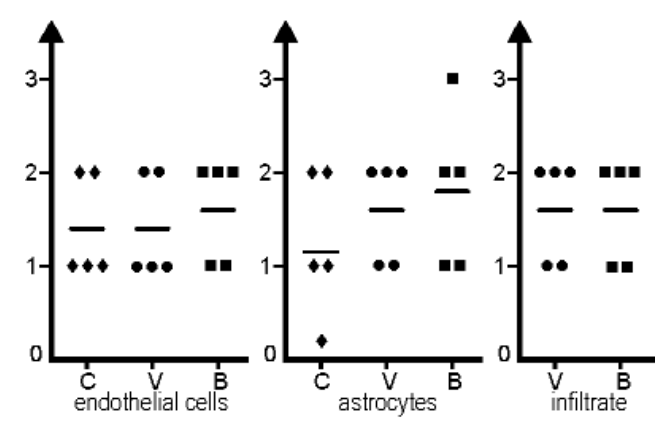

TIMP-2

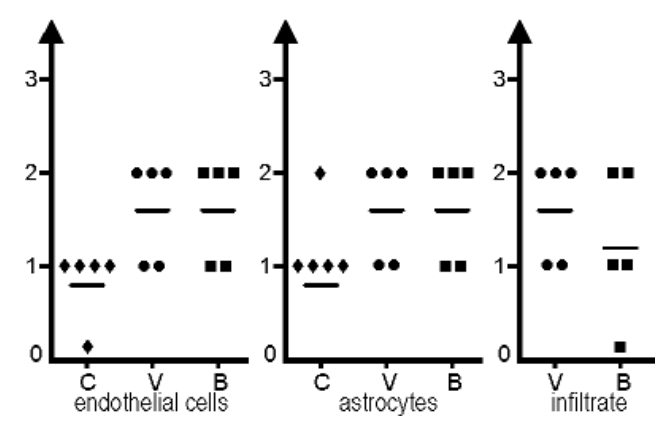

Fig. 1. Expression of MMP-2, MMP-9, TIMP-1 and TIMP-2 in endothelial cells, astrocytes and inflammatory infiltrates in samples collected from normal controls (C), as well as samples collected from patients with viral (V) and bacterial (B) meningitis. 0 , no immunoreactivity, 1, weak immunoreactivity, 2, moderate immunoreactivity, 3, intense immunoreactivity. Lines represent the mean value.
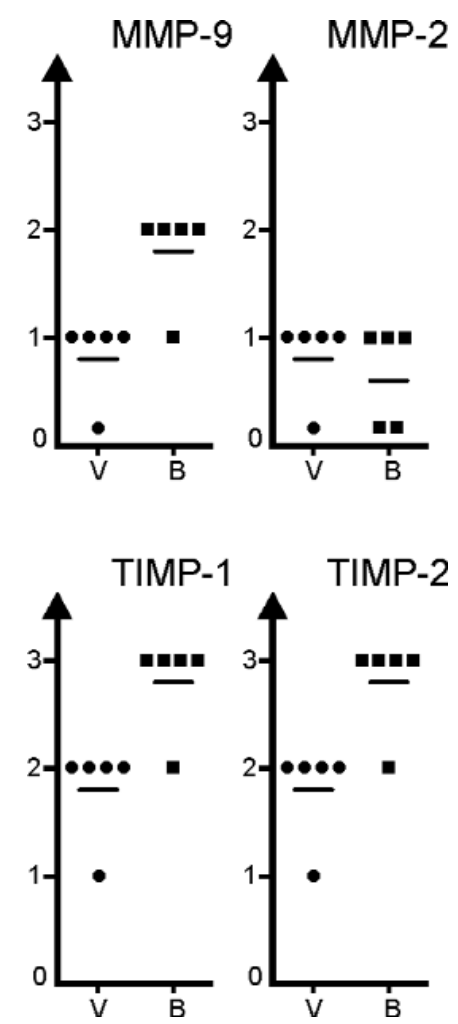

Fig. 2. Thickened meninges. Immunodetection of MMP-2, MMP9, TIMP-1 and TIMP-2 in infiltrating mononuclear cells in samples collected from patients with viral meningitis and polymorphonuclear cells in samples collected from patients with bacterial meningitis. Lines represent the mean value.

sue inhibitors (TIPM-1 and TIMP-2) in autopsy brain tissues obtained from patients with infectious inflammation to identify potential sources of their activity in the CNS.

The results of this study revealed that MMP-2 was immunolocalized in both normal controls and meningitis samples in brain parenchyma. Control samples and meningitis samples showed the same level of MMP-2 expression. In cantrast, MMP-9 immunoreactivity in brain endothelial cells, a key component of the blood-brain barrier, was higher in samples collected from patients with meningitis than in samples collected from control patients. These findings suggest that MMP-9 is crucially involved in the pathogenesis of meningitis, but that MMP-2 may not be, which is in accordance with the results of other studies. For example, in cases of tuberculous meningoencephalitis, MMP-9 was visualized in inflammatory infiltrates in the brain parenchyma, but MMP-2 was not, even though they were both present in infiltrating mononuclear cells [11]. In that study, MMP-2 was also detected in every cerebrospinal fluid (CSF) sample evaluated while MMP-9 was only detected in samples collected from patients with inflammatory diseases when measured using an enzyme immunoassay. Furthermore, in a study that evaluated children with bacterial 

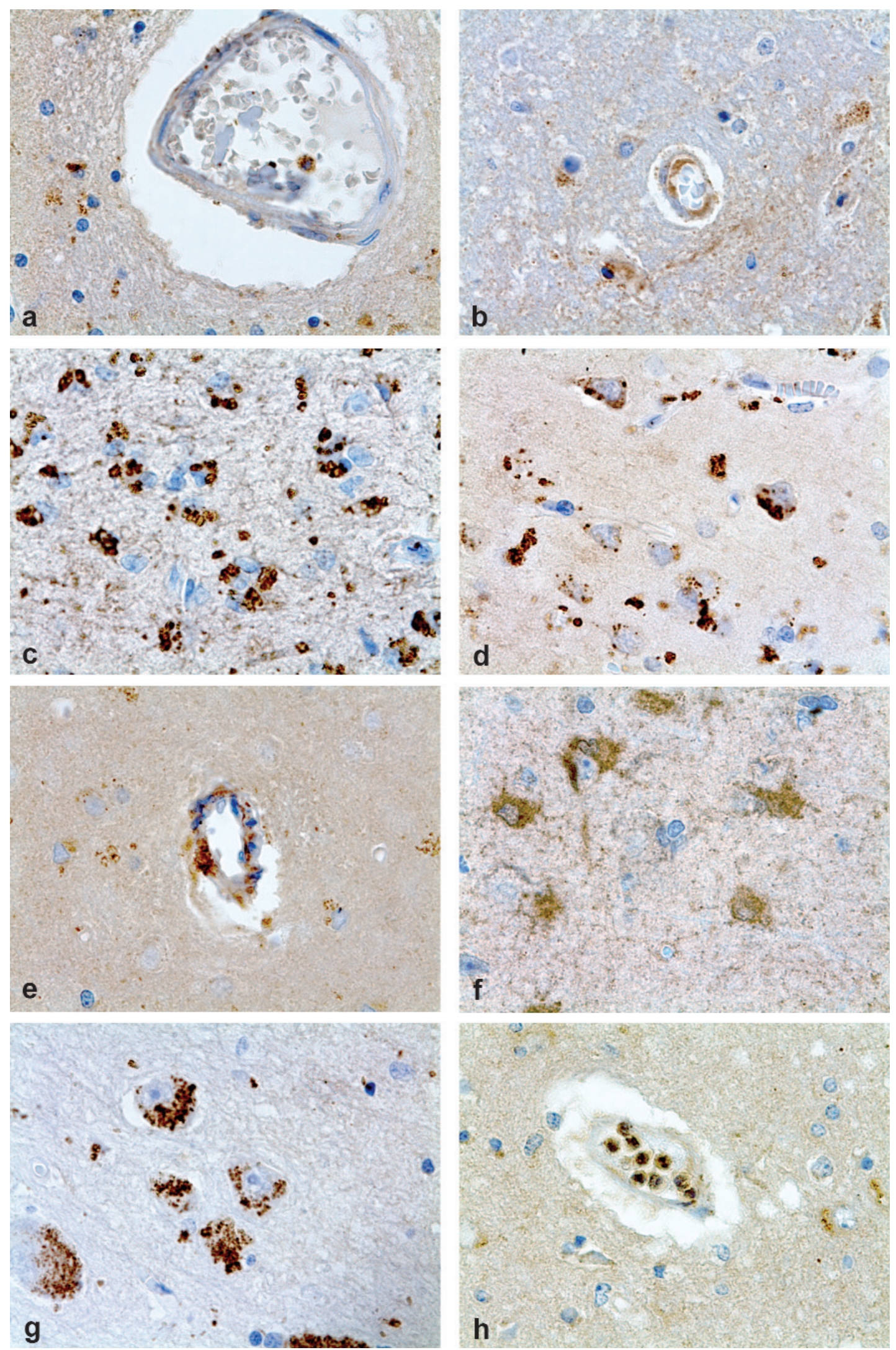

Fig. 3. Matrix metalloproteinase-9 (MMP-9) staining: endothelial layer in normal controls (a) and increased expression in samples obtained from patients with viral meningitis (b). Tissue inhibitor of metalloproteinase-1 (TIMP-1): increased expression was observed in astrocytes from meningitis patients (c) when compared to those from normal controls (d). MMP-2 immunolocalized in endothelial cells from viral meningitis (e). MMP-2 (f) and TIMP-2 (g) positive neurons. Strong MMP-9 immunoreactivity in polymorphomuclear cells (h) in purulent meningoencephalitis (magnification $\times 400$ ). 
meningitis, both gelatinases were detected in the CSF, while only MMP-2 was observed in CSF samples collected from controls [12]. However, detectable MMP9 levels have been found in CSF samples from some patients with viral meningitis when they were not present in samples collected from controls, and these levels were found to be correlated with CSF pleocytosis. In addition, in cases of viral meningitis, MMP-2 has been shown to be constantly expressed, but not upregulated [13]. Direct interaction with an etiological agent during meningitis could explain this increased MMP activity. Indeed, treatment with heat-inactivated Streptococcus pneumoniae was found to stimulate the release of MMP in brain endothelial cells [14]. Taken together, the results of the present study and previous findings demonstrate that MMP-9 plays a key role in CNS inflammation.

In this study, immunohistochemistry revealed that the expression pattern of the evaluated MMPs and TIPMs varied depending on cell type. Astrocytes appear to be crucially involved in the overall regulation of MMP activity. Interestingly, we found a decrease in MMP-9 immunoreactivity in astrocytes in samples collected from patients with meningitis and increase in TIMP-1 immunoreactivity under the same conditions. These findings may indicate that MMPTIMP complexes form during inflammation. Astrocytes are an important source of TIMP activity [15, 16], and a net balance between MMPs and TIMPs may be involved in the pathogenesis of CNS disease [17]. In addition to inhibiting MMPs, TIMPs are multifunctional molecules that have distinct biological roles [18]. Furthermore, like MMPs, TIMPs induce both beneficial and detrimental effects [19].

Analysis from serial lumbar punctures [7] revealed that the concentration of MMPs in the cerebrospinal fluid are only partially correlated with the CSF leukocyte count. This suggests that invading immune cells are only partially responsible for the activity of MMPs in the CSF during meningitis; therefore, parenchymal cells may be another relevant source. In this study, we found neurons to be a stable source of MMPs and TIPMs in the CNS in meningitis patients and normal controls.

The results of this study revealed that MMP-9 and TIMP-1 was much more immunoreactive in inflammatory infiltrates of meninges from patients afflicted with bacterial meningitis when compared to patients afflicted with viral meningitis. In a study of patients with tuberculosis meningitis, both MMP-2 and MMP-9 were immunolocalized to infiltrating mononuclear cells in the meninges but TIMP-1 and TIMP-2 were expressed in only a small number of inflammatory cells in thickened meninges [11]. However, positive signals for MMP-9 and TIPM-1 were shown to be localized in infiltrating polymorphonu- clear cells and mononuclear cells in mice that had eosinophilic meningitis [20].

Understanding the processes that lead to brain damage during meningitis is crucial for the development of future drugs and the improvement of treatment protocols. Previous studies have generated encouraging data regarding the modulation of MMPs during CNS repair following injury. For example, inhibition of MMPs in rats was shown to prevent kainic acid-induced cell death in the brain, which suggests that MMP inhibitors have some therapeutic potential [21]. Furthermore, inhibition of MMPs using a broad-spectrum metalloproteinase inhibitor ameliorated neutrophil infiltration following intracerebral haemorrhage and induced function improvement and a decrease in the volume of injury [22]. It is likely that regulating MMP activity could enable beneficial effects in the treatment of neuroinflammation as well; however, further investigation focused on specific target cells in the central nervous system is required.

\section{References}

[1] Avolio C, Ruggieri M, Giuliani F, et al. Serum MMP-2 and MMP-9 are elevated in different multiple sclerosis subtypes. J Neuroimmunol. 2003;136:46-53.

[2] Nygardas PT, Hinkkanen AE. Up-regulation of MMP-8 and MMP-9 activity in the BALB/c mouse spinal cord correlates with the severity of experimental autoimmune encephalomyelitis. Clin Exp Immunol. 2002;128:245-54.

[3] Lorenzl S, Albers DS, Relkin N, et al. Increased plasma levels of matrix metalloproteinase-9 in patients with Alzheimer's disease. Neurochem Int. 2003;43:191-196.

[4] Rosell A, Ortega-Aznar A, Alvarez-Sabín J, et al. Increased brain expression of matrix metalloproteinase-9 after ischemic and hemorrhagic human stroke. Stroke. 2006;37:1399-1406.

[5] Lee KY, Kim EH, Yang WS, et al. Persistent increase of matrix metalloproteinases in cerebrospinal fluid of tuberculous meningitis. J Neurol Sci. 2004;220:73-78.

[6] Sellner J, Leib SL. In bacterial meningitis cortical brain damage is associated with changes in parenchymal MMP9/TIMP-1 ratio and increased collagen type IV degradation. Neurobiol Dis. 2006;21:647-656.

[7] Lindberg RL, Sorsa T, Tervahartiala T, et al. Gelatinase B [matrix metalloproteinase (MMP)-9] and collagenases (MMP-8/-13) are upregulated in cerebrospinal fluid during aseptic and bacterial meningitis in children. Neuropathol Appl Neurobiol. 2006;32:304-317.

[ 8] Yushchenko M, Weber F, Mäder M, et al. Matrix metalloproteinase-9 (MMP-9) in human cerebrospinal fluid (CSF): elevated levels are primarily related to CSF cell count. $J$ Neuroimmunol. 2000;110:244-251.

[9] Yong VW. Metalloproteinases: mediators of pathology and regeneration in the CNS. Nat Rev Neurosci. 2005;6:931-944.

[10] Chirco R, Liu XW, Jung KK, Kim HR. Novel functions of TIMPs in cell signaling. Cancer Metastasis Rev. 2006;25:99113.

[11] Matsuura E, Umehara F, Hashiguchi T, Fujimoto N, Okada Y, Osame M. Marked increase of matrix metalloproteinase 9 in cerebrospinal fluid of patients with fungal or tuberculous meningoencephalitis. J Neurol Sci. 2000;173:45-52. 
[12] Shapiro S, Miller A, Lahat N, Sobel E, Lerner A. Expression of matrix metalloproteinases, sICAM-1 and IL-8 in CSF from children with meningitis. J Neurol Sci. 2003;206:43-48.

[13] Kolb SA, Lahrtz F, Paul R, et al. Matrix metalloproteinases and tissue inhibitors of metalloproteinases in viral meningitis: upregulation of MMP-9 and TIMP-1 in cerebrospinal fluid. J Neuroimmunol. 1998;84:143-150.

[14] Michel U, Zobotke R, Mäder M, Nau R. Regulation of matrix metalloproteinase expression in endothelial cells by heatinactivated Streptococcus pneumoniae. Infect Immun. 2001;69:1914-1916.

[15] Giraudon P, Szymocha R, Buart S, et al. T lymphocytes activated by persistent viral infection differentially modify the expression of metalloproteinases and their endogenous inhibitors, TIMPs, in human astrocytes: relevance to HTLV-Iinduced neurological disease. J Immunol. 2000;164:27182727.

[16] Gardner J, Borgmann K, Deshpande MS, et al. Potential mechanisms for astrocyte-TIMP-1 downregulation in chronic inflammatory diseases. J Neurosci Res. 2006;83:1281-1292.

[17] Gardner J, Ghorpade A. Tissue inhibitor of metalloproteinase (TIMP)-1: the TIMPed balance of matrix metalloproteinases in the central nervous system. J Neurosci Res. 2003;74:801-806.
[18] Brew K, Dinakarpandian D, Nagase H. Tissue inhibitors of metalloproteinases: evolution, structure and function. Biochim Biophys Acta. 2000;1477:267-283.

[19] Candelario-Jalil E, Yang Y, Rosenberg GA. Diverse roles of matrix metalloproteinases and tissue inhibitors of metalloproteinases in neuroinflammation and cerebral ischemia. Neuroscience. 2008 doi:10.1016/j.neuroscience.2008.06.025

[20] Chen KM, Lee HH, Chou HL, Liu JY, Tsai BC, Lai SC. Upregulation of MMP-9/TIMP-1 enzymatic system in eosinophilic meningitis caused by Angiostrongylus cantonensis. Int J Exp Pathol. 2005;86:81-89.

[21] Campbell SJ, Finlay M, Clements JM, Wells G, Miller KM, Perry VH, Anthony DC. Reduction of excitotoxicity and associated leukocyte recruitment by a broad-spectrum matrix metalloproteinase inhibitor. J Neurochem. 2004;89:13781386.

[22] Wang J, Tsirka SE. Neuroprotection by inhibition of matrix metalloproteinases in a mouse model of intracerebral haemorrhage. Brain. 2005;128:1622-1633.

Submitted: 15 August, 2008 Accepted after reviews: 26 September, 2008 